\title{
O Problema do Poder Efetivo na Câmara dos Deputados
}

\author{
Renan Barbosa de Morais \\ Universidade Federal de Itajubá \\ Itajubá, MG, Brasil \\ renan@unifei.edu.br \\ Mário César San Felice \\ Universidade Federal de São Carlos \\ São Carlos, SP, Brasil \\ felice@ufscar.br
}

\author{
Pedro Henrique Del Bianco Hokama \\ Universidade Federal de Itajubá \\ Itajubá, MG, Brasil \\ hokama@unifei.edu.br \\ Gabriel Ávila Casalecchi \\ Universidade Federal de São Carlos \\ São Carlos, SP, Brasil \\ gacasalecchi@gmail.com
}

\begin{abstract}
Proportionality in political representation is an essential theme for representative democracy. In Brazil, this debate appears in the context of non-proportionality between a federative unit's population size and its number of representatives in the Chamber of Deputies. In other words, the number of deputies in a state is not proportional to its number of inhabitants, which violates the "one man, one vote" principle.

Discussions around this disproportionality have motivated scholars to develop empirical research that aims to identify the causes and consequences of the phenomenon and to analyze the impact that the rule introduces in the political process. This article seeks to contribute to this debate by measuring the effective power of each Brazilian federation's entity and proposing alternatives of distribution for the Brazilian Chamber of Deputies.

To this end, we use a mathematical concept from game theory, called Power Index, which allows quantifying the existing representational discrepancies. After evaluating several distributions, we solved the Inverse Power Index Problem (IPIP) to obtain a distribution of chairs that reduces such disparities. To solve the IPIP, which is computationally hard, we use an evolutionary heuristic. As an objective function to minimize the discrepancy, we use the linear Shapley rule, in which the power index of each state is proportional to its population.
\end{abstract}

\section{KEYWORDS}

Problema de Índice de Potência Inversa, BRKGA, Democracia Representativa, Câmara dos Deputados

\section{INTRODUÇÃO}

A proporcionalidade na representação política é tema importante para a Ciência Política, particularmente para a literatura que pesquisa a democracia e os sistemas políticos. No Brasil, um dos principais pontos de debate é o da não-proporcionalidade entre a população de uma circunscrição eleitoral e o seu número de representantes na Câmara dos Deputados. Em outras palavras, o número de deputados de um estado não é exatamente proporcional ao número de habitantes, o que fere a máxima "uma cabeça, um voto".

Existem duas formas mais comuns de violação da proporcionalidade entre habitantes e representantes [1]: 1) a ausência de revisão periódica do número de representantes de cada circunscrição eleitoral em virtude de mudanças demográficas da população e 2) a inclusão de limites mínimos e máximos de deputados por circunscrição. A inclusão dos limites está no Art. $45, \S 1^{\circ}$, da Constituição Federal: "O número total de Deputados, bem como a representação por Estado e pelo Distrito Federal, será estabelecido por lei complementar, proporcionalmente à população, procedendo-se aos ajustes necessários, no ano anterior às eleições, para que nenhuma daquelas unidades da Federação tenha menos de oito ou mais de setenta Deputados."

O caso mais utilizado para ilustrar a distorção entre número de representantes e população é comparar os estados de São Paulo e Roraima. No primeiro, o número de habitantes por deputado é de quase 656 mil, enquanto no segundo é próximo de 76 mil pessoas. Ou seja, em São Paulo cada deputado representa aproximadamente 8,6 vezes mais pessoas que em Roraima (de acordo com a população em 2019 [2]). Essa disparidade tem dividido os estudiosos da Ciência Política. Por um lado, o principal argumento a favor de uma representação estritamente proporcional em termos de população e cadeiras parlamentares seria o seu caráter mais democrático. Como visto no exemplo acima, ele infringe diretamente o princípio igualitário da democracia, no qual cada indivíduo tem o mesmo valor em termos de voto.

Por outro lado, a principal objeção a esse argumento é que a democracia não se limita ao princípio da igualdade e a modelos majoritários de decisão. De acordo com Soares e Lourenço [3], é possível defender uma outra concepção "que privilegie a inclusão e o consenso, dando expressão a interesses relevantes presentes na sociedade, mesmo que minoritários”. A introdução de limites seria positiva, pois garantiria a representatividade efetiva de estados e populações minoritários, que de outra forma não teriam condições de fazer valer suas demandas $[4,5]$.

As discussões em torno da desproporcionalidade têm motivado estudiosos a desenvolver pesquisas empíricas que visem não só identificar as causas e consequências do fenômeno, como também analisar o impacto que a regra introduz no processo político [6, 7]. Preocupações semelhantes, envolvendo encontrar uma representação justa em um cenário com diversos agentes, motivaram estudos utilizando um conceito matemático da área de teoria dos jogos, chamado Power Index [8-10]. Em particular, diversos desses estudos são relacionados à composição do Conselho Europeu [11-13]. No Brasil, Lima e Ramos [14] utilizaram o conceito de power index para estudar os estados e regiões brasileiras, além de discutir as implicações econômicas.

O Power Index de um agente corresponde ao poder que o mesmo tem de afetar o resultado de uma votação de acordo com seu peso. 
Assim, é possível quantificar as discrepâncias de representatividade existentes. Tomemos como exemplo o Conselho da Comunidade Econômica Europeia, composto por Alemanha, França, Itália, Bélgica, Holanda e Luxemburgo. Cada um dos três primeiros países possuíam peso 4, Bélgica e Holanda tinham peso 2 cada e Luxemburgo tinha peso 1. Para alguma resolução ser aprovada, era necessário um total de 12. Embora esta distribuição de votos possa parecer justa e proporcional ao tamanho, poder ou população de cada país, Luxemburgo não possuía nenhuma influência nas decisões. Isto porque em qualquer situação seu voto não tinha o poder de alterar o resultado da votação. Isto é, qualquer coalizão que ganhava ou perdia sem Luxemburgo continuava a ganhar ou perder com Luxemburgo.

Intuitivamente, o valor dos power indices é baseado na capacidade que um agente tem para mudar o resultado de uma votação com o seu voto. Mas, qual é uma distribuição de power indices justa? Penrose [10] defende que para uma distribuição justa de poder, os agentes devem possuir power indices que sejam proporcionais à raiz quadrada de sua população. Muito difundida, esta abordagem foi proposta por Zyczkowski e Slomczynski [15] em uma reformulação do sistema de votação no Conselho Europeu. Kurz et al. [16] alegam que quando os agentes possuem uma grande polarização entre eles, para uma distribuição de poder mais justa, os power indices devem ser proporcionais à população, no que batizam de linear Shapley rule. Mas como saber o peso dos votos que resulta no poder efetivo desejado? Para isso precisamos resolver o Problema Inverso dos Power Indices (IPIP, do inglês Inverse Power Index Problem).

Contribuições. Neste trabalho, calculamos o poder efetivo (power index) de cada estado da federação brasileira considerando a atual configuração da Câmara dos Deputados, bem como uma distribuição proporcional à população em 2019, na qual os limitantes superior e inferior sejam abolidos. Então, desenvolvemos uma heurística evolutiva que usa o framework BRKGA (Biased Random-Key Genetic Algorithm) para resolver o IPIP. Usando nossa heurística, propomos uma distribuição baseada na linear Shapley rule, onde o poder efetivo de cada estado é proporcional a sua população. Destacamos que, para relacionar o peso do voto de um agente com o seu número de representantes, neste trabalho consideramos cada estado como um único agente. Isto é, supomos que os representantes de um estado atuam em bloco nas medidas que favoreçam sua unidade federativa. Além disso, ressaltamos que utilizamos nas nossas análises toda a população das unidades federativas, considerando que os congressistas tem por função representar e defender os interesses da população, não apenas daqueles que votaram neles, ou ainda daqueles que tem direito a voto. Até onde sabemos, este trabalho é o primeiro a resolver o IPIP a fim de encontrar uma distribuição mais justa para a Câmara dos Deputados brasileira.

Organização. A Seção 2 descreve o conceito de power index. Na Seção 3, é realizada uma análise dos poderes efetivos na Câmara dos Deputados brasileira. A Seção 4 apresenta o IPIP e a heurística evolutiva proposta. Na Seção 5 é apresentado a distribuição encontrada seguindo a linear shapley rule e, na Seção 6 são realizadas as considerações finais.

\section{POWER INDEX}

Em muitos problemas de tomada de decisão, alguma forma de votação é aplicada. Em algumas delas, os atores envolvidos possuem votos com pesos distintos. Esse tipo de votação, conhecida como jogo de votação ponderada, é usada em muitos cenários, como eleições, decisões de stakeholders em organizações e deliberações de instituições políticas.

Um jogo de votação ponderada possui um conjunto finito $N$ com $n$ jogadores e uma quota $q \in \mathbb{R}_{+}^{*}$. Cada jogador possui um peso $w \in \mathbb{R}_{+}$. Um subconjunto $S \subseteq N$ é chamado de coalizão e o ganho de uma coalizão $S$ é $v(S)$. $N$ é chamado de grande coalizão.

Como, nesse tipo de jogo, as coalizões somente ganham ou perdem, $v(S)=1$ se $S$ é uma coalizão vencedora e $v(S)=0$ se perdedora. O conjunto de coalizões vencedoras $W$ contém todas as coalizões onde $v(S)=1$. Ademais, uma coalizão vencedora não pode se tornar perdedora ao adicionar um jogador e de modo semelhante, uma coalizão perdedora não se torna vencedora ao se retirar um jogador.

Em um jogo de votação ponderada, os pesos $w_{1}, \ldots, w_{n}$ e a cota $q$ definem se uma coalizão é perdedora ou vencedora através de sua função caraterística $v: 2^{n} \rightarrow\{0,1\}$, onde:

$$
v(S)= \begin{cases}1 & \text { se } \sum_{i \in S} w_{i} \geq q \\ 0 & \text { caso contrário. }\end{cases}
$$

Uma das principais razões para o uso de jogos de votação ponderada é que cada ator possua uma representação justa ao decidir sobre algum assunto. Porém, muitas vezes o peso atribuído à eles não corresponde à influência real na votação. Como apresentado anteriormente, em geral o peso de um jogador não é equivalente ao seu poder efetivo. Diferentes power indices surgiram desta necessidade de se medir a influência de um jogador em um jogo. Dois dos principais power indices são o Banzhaf Power Index [17] e o Shapley-Shubik Power Index [18].

\subsection{Banzhaf power index}

O Banzhaf Power Index calcula a quantidade de coalizões em que um jogador é decisivo. Isto ocorre se uma coalização é vencedora com este jogador e deixa de ser retirando-o deste conjunto. O Banzhaf index $\beta_{i}^{\prime}$ é o número de coalizões em que o jogador $i$ é decisivo em um jogo e pode ser descrito como:

$$
\beta_{i}^{\prime}=|\{S \subseteq N-\{i\} \mid v(S)=0 \wedge v(S \cup\{i\})=1\}|
$$

O Banzhaf power index [19] é definido como:

$$
\beta_{i}=\frac{\beta_{i}^{\prime}}{\sum_{j=1}^{n} \beta_{j}^{\prime}}
$$

\subsection{Shapley-Shubik power index}

Seja $\Pi$ o conjunto de permutações de $n$ jogadores em um jogo, $\pi \in \Pi$ uma permutação e $\Upsilon_{i} \subseteq \pi$ o subconjunto que precede o jogador $i$ em $\pi$. Para cada $\pi \in \Pi$, um jogador $i$ é o pivô se ele é o primeiro a tornar a coalizão vencedora. Assim, $\varphi_{i}$ é a quantidade de vezes em que isso ocorre, dado por:

$$
\varphi_{i}=\sum_{\pi \in \Pi} v\left(\Upsilon_{i} \cup\{i\}\right)-v\left(\Upsilon_{i}\right)
$$


O termo $\varphi_{i}$ também é chamado de raw Shapley-Shubik index. O Shapley-Shubik Power Index do jogador $i$ é dado por $\varphi_{i} / n$ !. Uma maneira mais conveniente para calcular esse power index é:

$$
\phi_{i}=\sum_{S \notin W: S \cup i \in W} \frac{|S| !(|N|-|S|-1) !}{|N| !}
$$

sendo $W$ o conjunto das coalizões vitoriosas.

\section{PODER NA CÂMARA DOS DEPUTADOS}

Considerando a representação de cada unidade federativa na Câmara dos Deputados, se pode mensurar o poder efetivo de cada uma delas por meio dos power indices.

\subsection{Distribuição atual}

A Tabela 1 mostra o número atual de cadeiras para cada estado da federação. Note que, a atual distribuição leva em conta a população de 1993, conforme estabelecido na Lei Complementar $n^{\circ} 78$ de 30/12/1993.

A partir dos pesos, representados pelo número de deputados, e da cota, representado pelo número de 257 deputados necessários para aprovação de uma lei complementar, ou de um projeto de lei e medida provisória com quórum máximo, podemos calcular o poder efetivo de cada estado. A Fig. 1 apresenta a comparação entre o Shapley-Shubik power index de cada unidade federativa e seu peso normalizado.

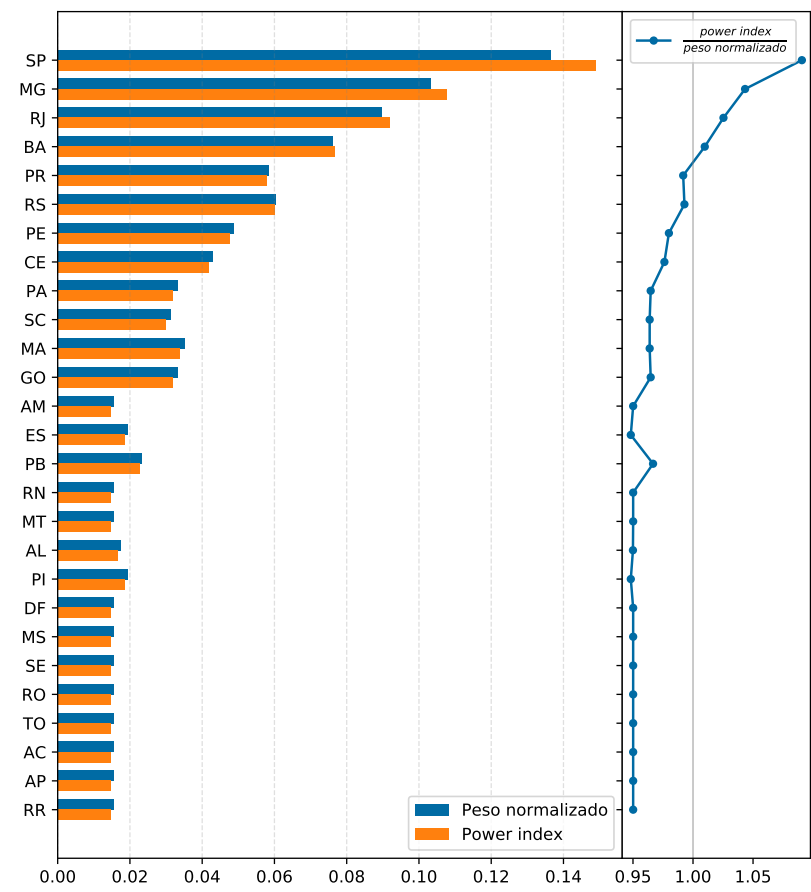

Figura 1: Comparação entre peso normalizado e ShapleyShubik power index na distribuição atual
Notamos que o número de representantes (peso) que cada estado possui não corresponde a seu poder efetivo. Os quatro estados com maior população (São Paulo, Minas Gerais, Rio de Janeiro e Bahia) possuem poder efetivo maior do que seu peso, enquanto todos os outros têm poder menor.

Podemos então comparar os power indices com a população, conforme ilustra a Fig. 2. Essa comparação nos mostra que apesar do estado de São Paulo possuir um poder efetivo superior a seu número de deputados normalizado, seu power index ainda é desproporcionalmente pequeno em relação a sua população. Por outro lado, os estados atingidos pelo piso apresentam um poder efetivo bem maior em relação a sua população.

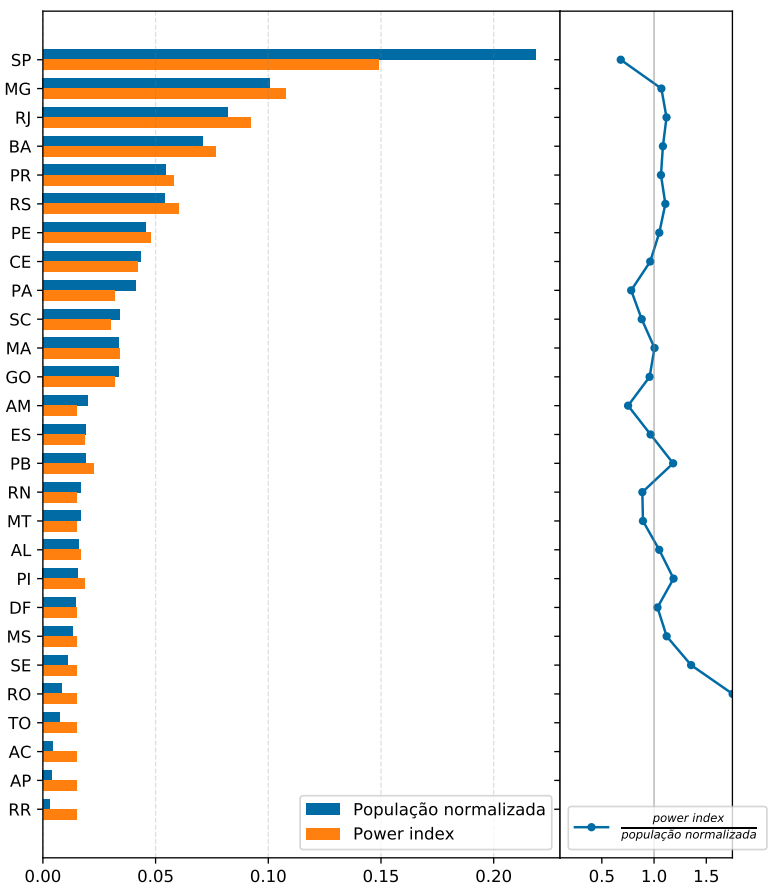

Figura 2: Comparação entre população normalizada e Shapley-Shubik power index na distribuição atual

Assim, notamos que a presença de um teto e um piso, com o objetivo de restringir a influência das unidades federativas com população muito grande e de ampliar a participação daquelas com população pequena causa diversas distorções. Será que, abolindo o teto e o piso conseguiríamos uma representação mais justa?

\subsection{Distribuição proporcional à população atual}

Uma das críticas frequentes ao atual sistema de distribuição é que os habitantes em São Paulo não valem tanto quanto os dos outros estados. Analisamos então uma distribuição em que o número de representantes é estritamente proporcional à população de cada estado, sem limitantes inferiores e superiores. Mostraremos que essa distribuição também possui problemas. Mantendo o número total 


\begin{tabular}{lcccclcc} 
Estado & \#Dep. & & Estado & \#Dep. & & Estado & \#Dep. \\
\cline { 1 - 2 } Acre & 8 & & Maranhão & 18 & & Rio de Janeiro & 46 \\
Alagoas & 9 & & Mato Grosso & 8 & & Rio Grande do Norte & 8 \\
Amapá & 8 & & Mato Grosso do Sul & 8 & & Rio Grande do Sul & 31 \\
Amazonas & 8 & & Minas Gerais & 53 & & Rondônia & 8 \\
Bahia & 39 & & Pará & 17 & & Roraima & 8 \\
Ceará & 22 & & Paraíba & 12 & & Santa Catarina & 16 \\
Distrito Federal & 8 & & Paraná & 30 & & São Paulo & 70 \\
Espírito Santo & 10 & & Pernambuco & 25 & & Sergipe & 8 \\
Goiás & 17 & & Piauín & 10 & & Tocantins & 8
\end{tabular}

Tabela 1: Distribuição atual de deputados na Câmara dos Deputados.

de 513 deputados, a Tabela 2 ilustra a quantidade de parlamentares por estado, considerando a população de 2019.

É importante observar que o produto da população normalizada por 513 não resulta em números exatos. Para resolver isso, arredondamos-os para o inteiro mais próximo. Ao arrendondar obtivemos um total de 512 cadeiras, e distribuímos a última cadeira ao estado que estava mais próximo de ser aproximado para cima, o estado de Santa Catarina.

Grande parte das mudanças se deve à diferença da população em 2019 comparada a de 1993. Como exemplo, os estados do Amazonas e da Paraíba, que possuem atualmente 8 e 12 deputados respectivamente, teriam atualizações em sentidos opostos, se igualando no número de 10 deputados.

Vemos que o estado de São Paulo com folga é o que mais possui alteração no número de deputados, ganhando 42 novas cadeiras. Os estados beneficiados pelo piso sofrem reduções significativas, com Roraima possuindo apenas uma cadeira nessa nova configuração. Mas será que essa distribuição ficou justa?

A Fig. 3 ilustra a relação entre o Shapley-Shubik power index de cada estado com sua população normalizada. Intuitivamente, a distribuição estritamente proporcional traria um cenário mais justo, igualando o poder dos cidadãos de cada estado. No entanto, analisando o poder efetivo vemos que ainda existe uma grande disparidade. Em particular, São Paulo possui aproximadamente $26,15 \%$ do poder enquanto sua população representa $21,85 \%$ da população brasileira. Em verdade, todos os outros estados têm poder efetivo inferior a suas populações.

Como o número de deputados é proporcional à população de cada estado, a relação entre peso e poder efetivo é semelhante à mostrada na Fig. 3, com pequenas divergências causadas por arredondamentos.

\subsection{Alternativas de distribuição}

Vimos que, atualmente a Câmara dos Deputados possui uma distribuição de cadeiras com muitas deturpações. Porém, uma proposta estritamente proporcional à população causaria distorções muito significativas no poder efetivo dos estados, principalmente em favor de São Paulo.

No parlamento existem várias propostas que, ao propor a redução do número de deputados, acabam alterando as distorções pelo teto e piso e corrigindo a desatualização populacional, como as PEC 12/2019, PEC 431/2018, PEC 405/2018 e PEC 106/2015, para

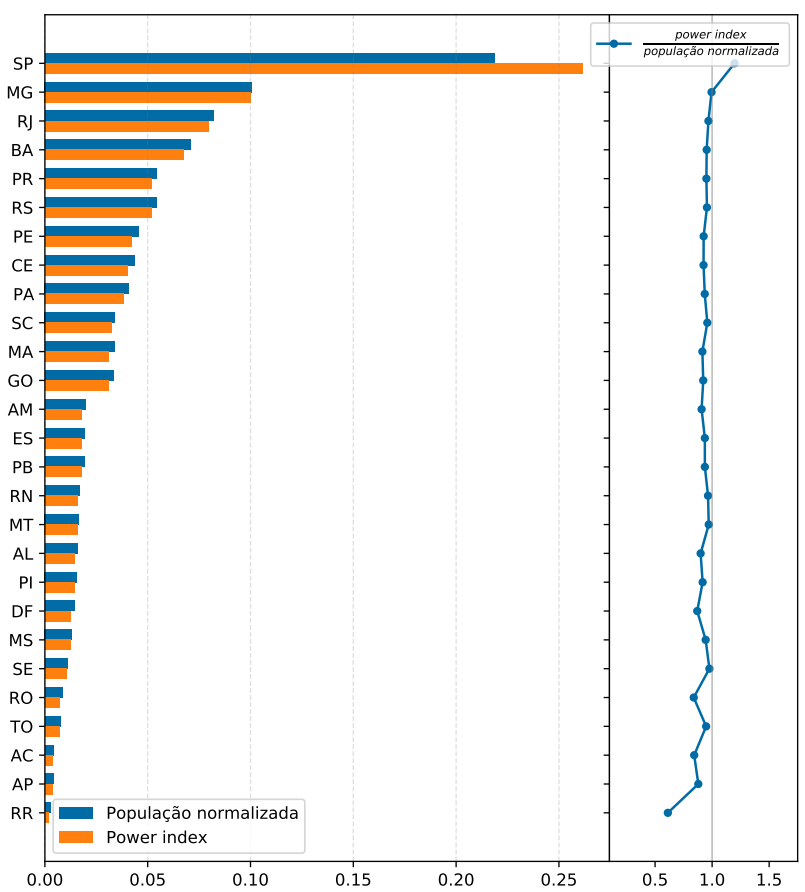

Figura 3: Comparação entre população normalizada e Shapley-Shubik power index na distribuição proporcional à população

citar alguns exemplos. Em relação à atualização populacional, a Resolução 23.389/2013 do Tribunal Superior Eleitoral redefiniu a distribuição de deputados considerando a população da época, porém acabou não vigorando após ser julgada inconstitucional pelo Supremo Tribunal Federal.

Existe, na literatura, diversas discussões sobre alternativas de distribuição na Câmara dos Deputados que seriam mais justas. Propostas baseadas na proporcionalidade regressiva [7], na penrose square root rule [14] ou até mesmo no aumento do número de deputados [6] são algumas das soluções propostas. No contexto dos power indices, a penrose square root rule é muito utilizada em cenários para obter uma distribuição de poder justa. Nessa regra, de forma 


\begin{tabular}{|c|c|c|c|c|c|c|c|c|}
\hline \multirow[b]{2}{*}{ Estado } & \multicolumn{2}{|c|}{ Diferença $\mathrm{p} /$} & \multicolumn{4}{|c|}{ Diferença $\mathrm{p} /$} & \multicolumn{2}{|c|}{ Diferença $\mathrm{p}$} \\
\hline & \#Dep. & Atual & Estado & \#Dep. & Atual & Estado & \#Dep. & Atual \\
\hline Acre & 2 & -6 & Maranhão & 17 & -1 & Rio de Janeiro & 42 & -4 \\
\hline Alagoas & 8 & -1 & Mato Grosso & 9 & +1 & Rio Grande do Norte & 9 & +1 \\
\hline Amapá & 2 & -6 & Mato Grosso do Sul & 7 & -1 & Rio Grande do Sul & 28 & -3 \\
\hline Amazonas & 10 & +2 & Minas Gerais & 52 & -1 & Rondônia & 4 & -4 \\
\hline Bahia & 36 & -3 & Pará & 21 & +4 & Roraima & 1 & -7 \\
\hline Ceará & 22 & & Paraíba & 10 & -2 & Santa Catarina & 18 & +2 \\
\hline Distrito Federal & 7 & -1 & Paraná & 28 & -2 & São Paulo & 112 & +42 \\
\hline Espírito Santo & 10 & & Pernambuco & 23 & -2 & Sergipe & 6 & -2 \\
\hline Goiás & 17 & & Piauí & 8 & -2 & Tocantins & 4 & -4 \\
\hline
\end{tabular}

Tabela 2: Distribuição de deputados proporcional à população em 2019, sem teto ou piso.

resumida, um agente deve ter uma probabilidade de ser decisivo em uma votação proporcional à raiz quadrada de sua população.

Propomos uma distribuição alternativa, que considera os problemas de poder efetivo já apresentados, que considera que, o que deve ser equivalente entre os cidadãos é o poder efetivo obtido a partir dos representantes eleitos, o qual muitas vezes difere significativamente do número de representantes de cada estado. De fato, nos baseamos na linear Shapley rule [16], onde uma distribuição justa se dá quando os power indices são proporcionais a população. $\mathrm{Ou}$ seja, queremos que o power index de cada estado seja proporcional a sua população. Desse modo, precisamos encontrar o número de deputados (peso) que cada estado deve possuir para atingir esse poder efetivo.

\section{PROBLEMA INVERSO E NOSSA HEURÍSTICA}

Para encontrar pesos que correspondem aos power indices desejados, precisamos resolver o Problema Inverso dos Power Indices. Seja $n$ o número de jogadores e $D=\left\{d_{1}, \ldots, d_{n}\right\}$ o conjunto de power indices desejado, com $d_{i} \geq 0$ e $\sum_{i=1}^{n} d_{i}=1$. Queremos encontrar um conjunto de pesos $W=\left\{w_{1}, \ldots, w_{n}\right\}$ e uma quota $q$, com $q>0$ e $w_{i} \geq 0$, onde minimizamos $\left|p_{i}-d_{i}\right|$, para todo jogador $1 \leq i \leq n$, na qual cada $p_{i}$ representa o power index do jogador de peso $w_{i}$.

Existem soluções propostas para o problema inverso que obtêm soluções sem garantia de otimalidade [20-22]. Até onde sabemos, somente dois autores propõem algoritmos exatos [23, 24]. Note que, uma solução ótima para esse problema corresponde a uma configuração que leva aos power indices desejados ou, se estes não são alcançáveis, que minimiza a distância até eles.

Propomos uma heurística evolutiva baseada no framework BRKGA, que encontra soluções para instâncias do IPIP de tamanho razoável. Destacamos que resolver o problema inverso é particularmente difícil, pois mesmo para avaliar a qualidade de uma solução, ou seja, calcular o power index de uma das distribuição de pesos, é preciso resolver um problema NP-difícil.

\subsection{Heurística evolutiva}

A ideia principal de um algoritmo evolutivo pode ser enunciado como: dada uma população de vários indivíduos, são selecionados dentre eles os que são mais aptos, que geram descendentes e integram uma nova população. Para isso, através de uma função para ser maximizada, pode-se mensurar a aptidão, também chamada de fitness, de cada indivíduo.

Nossa heurística é baseada no framework BRKGA, implementado por Toso e Resende [25]. De modo resumido, o framework gera aleatoriamente cromossomos, cujos alelos tem valor no intervalo $[0,1)$, evolui a população através de um crossover enviesado em favor dos mais aptos (elite) e insere cromossomos mutantes.

Precisamos decodificar os cromossomos afim de resolver nosso problema de otimização: o Problema Inverso. Para nosso cenário, os alelos de um cromossomo são, em um primeiro momento, equivalentes ao peso $w$ de cada jogador $i$. A quota $q$ é calculada somando o valor total e dividindo-o pela metade. Em seguida, é calculado o Shapley-Shubik power index de cada jogador, utilizando seu peso e a quota.

Para calcular o fitness de cada indivíduo, calcula-se a soma das diferenças entre o power index desejado e o power index encontrado através do cromossomo, dado por:

$$
\sum_{i=1}^{n}\left|d_{i}-p_{i}\right|
$$

O algoritmo inicia com uma população de $n$ cromossomos, cada um com $i$ alelos, que evolui até atingir um critério de parada. Para cada geração $k$, são executados os seguintes passos:

(1) Se calcula o fitness de cada indivíduo através da decodificação descrita anteriormente.

(2) A população é dividida em dois grupos: uma elite contendo $p_{e}$ indivíduos com os melhores valores de aptidão e uma não-elite com os indivíduos restantes.

(3) A geração $k+1$ será composta de:

(a) $p_{e}$ indivíduos da elite copiados da geração $k$.

(b) $p_{m}$ cromossomos gerados aleatoriamente (mutantes).

(c) $p_{o}=p-p_{m}-p_{e}$ descendentes produzidos através do cruzamento entre dois pais da geração $k$.

O cruzamento se dá selecionando aleatoriamente dois pais da geração atual, um da elite e um da não-elite. Para definir o $i$-ésimo alelo de um descendente, é gerado um número uniformemente aleatório $r \in[0,1)$. Se $r \leq \rho_{e} \operatorname{com} \rho_{e}>0,5$, o descendente herda o alelo $i$ do pai elite; caso contrário, herda o alelo $i$ do pai não-elite.

Ao final da execução, se tem um indivíduo de melhor fitness que contém o conjunto de pesos $W$. 


\section{UMA DISTRIBUIÇÃO LINEAR DE SHAPLEY}

Intuitivamente, pode-se argumentar que uma distribuição de poder mais justa se dá quando os power indices, e não os pesos, são proporcionais à população.

Na literatura, a Penrose square root rule [10] é objeto de estudo de muitos autores em diferentes cenários [8, 14, 15, 26]. Penrose [10] argumenta que em um sistema binário de decisão, isto é, os votantes apenas votam sim ou não, e onde todos os indivíduos possuem decisões estatisticamente independentes e igualmente prováveis, um agente deve ter uma probabilidade de ser pivô proporcional à raiz quadrada de sua população.

Kurz et al. [16] extendem a análise binária para um cenário de decisões onde os agentes possuem preferências em um intervalo. Isto é importante pois as decisões binárias não englobam conjunturas onde possa haver negociação e barganha. No contexto da Câmara dos Deputados isto é de fundamental importância por exemplo, em situações onde é discutido a distribuição de recursos para as unidades federativas.

Os autores chegam à conclusão que, em cenários onde há um alto nível de heterogeneidade nos interesses dos agentes, uma distribuição linear é mais adequada, a qual alcunham de linear Shapley rule. Nesse trabalho, estamos particularmente interessados nas votações em que os deputados decidem de forma homogênea dentro de suas UFs, e de forma heterogênea entre UFs. Em virtude da homogeneidade interna, podemos aproximar o número de deputados por um peso. Além disso, a heterogeneidade entre unidades federativas torna adequada a escolha da linear Shapley rule.

Então, encontramos uma distribuição para a Câmara dos Deputados na qual cada estado possui poder efetivo proporcional a sua população. Para isso, resolvemos o IPIP usando nossa heurística evolutiva, obtendo uma solução com erro 0,0095140. É importante observar que aqui também é preciso arrendondar o resultado, assim como nos outros cenários. Os números de deputados para as unidades federativas estão ilustrados na Tabela 3.

A proporção de cadeiras se mantém parecida com o caso estritamente proporcional. Apenas São Paulo possui uma diferença expressiva, com os outros estados tendo uma mudança de no máximo uma cadeira.

Agora, comparando o poder efetivo com a população em cada UF, obtemos uma diferença muito pequena, causada pelo erro encontrado na solução e, principalmente, pelo arredondamento. A Fig. 4 permite observar essa comparação, bem como notar que a distribuição proposta corrige tanto as distorções da distribuição atual quanto da estritamente proporcional.

Podemos comparar ainda a razão entre a população normalizada de cada estado com seu respectivo power index, para cada cenário analisado, como mostra a Fig. 5. Note que a solução encontrada possui razão mais próxima a 1 em relação aos outros cenários, principalmente se confrontado com a distribuição atual.

É importante observar que, conforme a população decresce, há maior dificuldade em a igualar com o power index. Isso ocorre por conta do arredondamento, que afeta o número de deputados e, consequentemente, o power index.

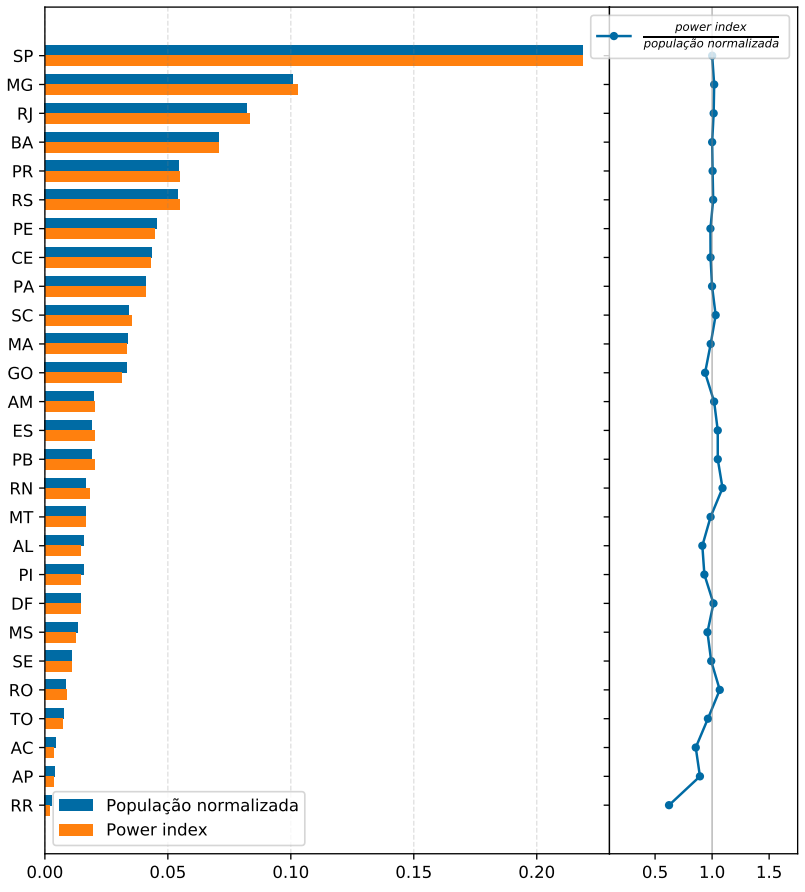

Figura 4: Comparação entre população normalizada e Shapley-Shubik power index na distribuição proposta

\section{CONSIDERAÇÕES FINAIS E TRABALHOS FUTUROS}

O power index de cada estado no sistema atual de proporcionalidade apresenta grande distorção em relação a sua população, devido à presença dos limitantes superiores e inferiores. Analisando um sistema onde não há limites demonstramos que, embora os estados afetados pelo piso passem a ter um poder efetivo próximo a sua população, o estado de São Paulo passa a ter poder efetivo muito superior às outras UFs.

Uma distribuição baseada na linear shapley rule encontrada resolvendo o IPIP aproxima os power indices dos estados de sua população, proporcionando uma alocação mais justa. Neste caso, o arredondamento causa algumas distorções, principalmente nos estados de menor população, mas menores do que no cenário anterior.

Embora a solução encontrada seja satisfatória, ainda há espaço para aprimoramento, reduzindo a distância entre os power indices e a população normalizada. O estudo de outras propostas de distribuição de poder pode trazer melhor discussão para este problema, dado que existem poucas investigações sobre o tema. Além disso, também parece interessante analisar o poder efetivo das regiões brasileiras.

Ademais, analisar eleições anteriores com as diferentes propostas de distribuição pode resultar em descobertas sobre a representação eleitoral nos estados sub e sobre-representados tanto do ponto de vista partidário como parlamentar, principalmente naqueles estados afetados pelo piso. 


\begin{tabular}{|c|c|c|c|c|c|c|c|c|c|c|c|}
\hline \multirow[b]{2}{*}{ Estado } & \multicolumn{3}{|c|}{ Diferença p/ } & \multicolumn{5}{|c|}{ Diferença p/ } & \multicolumn{3}{|c|}{ Diferença p/ } \\
\hline & \#Dep. & Atual & Prop. & Estado & \#Dep. & Atual & Prop. & Estado & \#Dep. & Atual & Prop. \\
\hline Acre & 2 & -6 & & Maranhão & 18 & & +1 & Rio de Janeiro & 43 & -3 & +1 \\
\hline Alagoas & 8 & -1 & & Mato Grosso & 9 & +1 & & Rio Grande do Norte & 10 & +2 & +1 \\
\hline Amapá & 2 & -6 & & Mato Grosso do Sul & 7 & -1 & & Rio Grande do Sul & 29 & -2 & +1 \\
\hline Amazonas & 11 & +3 & +1 & Minas Gerais & 52 & -1 & & Rondônia & 5 & -3 & +1 \\
\hline Bahia & 37 & -2 & +1 & Pará & 22 & +5 & +1 & Roraima & 1 & -7 & \\
\hline Ceará & 23 & +1 & +1 & Paraíba & 11 & -1 & +1 & Santa Catarina & 19 & +3 & +1 \\
\hline Distrito Federal & 8 & & +1 & Paraná & 29 & -1 & +1 & São Paulo & 97 & +27 & -15 \\
\hline Espírito Santo & 11 & +1 & +1 & Pernambuco & 24 & -1 & +1 & Sergipe & 6 & -2 & \\
\hline Goiás & 17 & & & Piauí & 8 & -2 & & Tocantins & 4 & -4 & \\
\hline
\end{tabular}

Tabela 3: Distribuição de deputados obtidos resolvendo o IPIP baseado na linear Shapley rule.

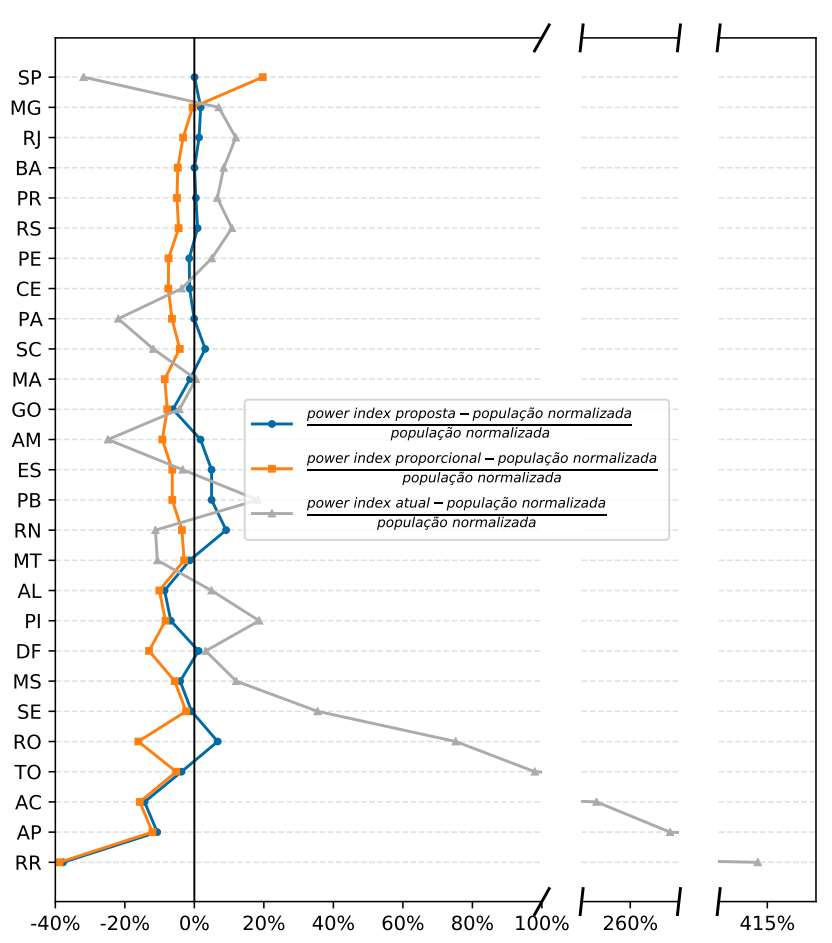

Figura 5: Comparação da razão entre o Shapley-Shubik power index e a população normalizada nos três cenários descritos anteriormente

É importante ressaltar que nossa heurística pode solucionar o problema inverso tanto para o Shapley-Shubik Power Index (utilizado na distribuição proposta) quanto para o Banzhaf Power Index. De modo semelhante, nossa abordagem se adéqua facilmente a regras diferentes da linear Shapley rule, como a penrose square root rule. Essa flexibilidade permite comparar o desempenho do nosso método com distribuições propostas por outros trabalhos, como Zyczkowski e Slomczynski [15] e Kurz [23]. Pretendemos realizar essas comparações em trabalhos futuros.

\section{AGRADECIMENTOS}

Apoiado por CNPq 435617/2018-4.

\section{REFERÊNCIAS}

[1] Jairo Marconi Nicolau. As distorções na representação dos estados na câmara dos deputados brasileira. Dados, 40(3), 1997.

[2] IBGE. Tabelas de estimativas populacionais para os municípios e para as unidades da federação brasileiros em 01.07.2019. ftp://ftp.ibge.gov.br/Estimativas_de_ Populacao/Estimativas_2019/estimativa_dou_2019.ods, 2019. Acessado em 04 de Junho de 2020 .

[3] Márcia Miranda Soares e Luiz Cláudio Lourenço. A representação política dos estados na federação brasileira. Revista Brasileira de Ciências Sociais, 19(56): 113-127, 2004.

[4] Preston King. Federation and representation. Comparative Federalism and Federation, 95, 1993.

[5] Arend Lijphart. Patterns of democracy: Government forms and performance in thirty-six countries. Yale University Press, 2012.

[6] Arlindo Fernandes de Oliveira. Sobre a representação dos Estados na Câmara dos Deputados. Senado Federal, Consultoria Legislativa, 2004.

[7] Erinaldo Ferreira Carmo, Enivaldo Carvalho Rocha, e Dalson Britto Figueiredo Filho. Aplicando o modelo de proporcionalidade degressiva na representação dos estados na câmara dos deputados. Revista do Direito Público, 7(3):191-204, 2012.

[8] Nicholas R Miller. A priori voting power and the us electoral college. In Power, Voting, and Voting Power: 30 Years After, pages 411-442. Springer, 2013.

[9] John F Banzhaf. Multi-member electoral districts. do they violate the"one man, one vote"principle. The Yale Law fournal, 75(8):1309-1338, 1966.

[10] Lionel S Penrose. The elementary statistics of majority voting. Fournal of the Royal Statistical Society, 109(1):53-57, 1946.

[11] Dennis Leech. Designing the voting system for the council of the european union. Public Choice, 113(3-4):437-464, 2002.

[12] Dan S Felsenthal e Moshé Machover. The treaty of nice and qualified majority voting. Social Choice and Welfare, 18(3):431-464, 2001.

[13] Annick Laruelle e Mika Widgrén. Is the allocation of voting power among eu states fair? Public Choice, 94(3-4):317-339, 1998.

[14] Ana Carolina da Cruz Lima e Francisco de Sousa Ramos. Há desigualdade de poder entre os estados e regiões do brasil? uma abordagem utilizando o índice de poder de banzhaf e a penrose square root law. Economia Aplicada, 14(2):225-249, 2010.

[15] Karol Zyczkowski e Wojciech Slomczynski. Voting in the european union: The square root system of penrose and a critical point. arXiv preprint condmat/0405396, 2004.

[16] Sascha Kurz, Nicola Maaser, e Stefan Napel. On the democratic weights of nations. Journal of Political Economy, 125(5):1599-1634, 2017.

[17] John F Banzhaf III. Weighted voting doesn't work: A mathematical analysis. Rutgers L. Rev., 19:317, 1964.

[18] Lloyd S Shapley e Martin Shubik. A method for evaluating the distribution of power in a committee system. American political science review, 48(3):787-792, 1954.

[19] Pradeep Dubey e Lloyd S Shapley. Mathematical properties of the banzhaf power index. Mathematics of Operations Research, 4(2):99-131, 1979.

[20] Shaheen Fatima, Michael Wooldridge, e Nicholas R Jennings. An anytime approximation method for the inverse shapley value problem. In Proceedings of the 7th international joint conference on Autonomous agents and multiagent systemsVolume 2, pages 935-942. International Foundation for Autonomous Agents and Multiagent Systems, 2008. 
XII Computer on the Beach

7 a 9 de Abril de 2021, Online, SC, Brasil

Morais et al.

[21] Haris Aziz, Mike Paterson, e Dennis Leech. Efficient algorithm for designing weighted voting games. In 2007 IEEE International Multitopic Conference, pages 1-6. IEEE, 2007.

[22] Dennis Leech. Computation of power indices. 2002

[23] Sascha Kurz. On the inverse power index problem. Optimization, 61(8):989-1011, 2012

[24] B De Keijzer. On the design and synthesis of voting games: Exact solutions for the inverse problems. 2009.
[25] Rodrigo F Toso e Mauricio GC Resende. A c++ application programming interface for biased random-key genetic algorithms. Optimization Methods and Software, 30(1):81-93, 2015.

[26] Dan S Felsenthal e Moshé Machover. Analysis of qm rules in the draft constitution for europe proposed by the european convention, 2003. Social Choice and Welfare, 23(1):1-20, 2004. 